\title{
Intraoperative MRT in der Neurochirurgie
}

\author{
Michal Hlavac, Ralph König, Rainer Wirtz
}

\section{Zusammenfassung}

Intraoperative Magnetresonanztomografie (iMRT) findet eine zunehmende Anwendung in der Resektion glialer Tumoren des Gehirns. Durch die iMRT ist es möglich, die intraoperativ auftretenden Veränderungen des OP-Situs in der besten Darstellungsmodalität zu erfassen und dem Operateur mithilfe der Neuronavigation situationsgerecht zu präsentieren. Zum Betrieb einer solch komplexen Technik ist ein hoher Grad an fachlicher Kompetenz aller beteiligten OP-Mitarbeiter erforderlich. Durch zunehmende Systemintegration und neue bildgebende Modalitäten der Hochfeldscanner eröffnen sich neue Perspektiven in Bezug auf die Effizienz und das Risikoprofil des chirurgischen Eingriffs. Die Auswertung eigener Daten zeigt eine deutliche Zunahme der vollständigen Tumorresektionen sowohl für die glialen Tumoren als auch für die Hypophysenadenome.

\section{Intraoperative MRI in Neurosurgery}

The application of intraopeartive magnetic resonance imaging (iMRI) in the resection of glial tumors is experiencing growing acceptance. The iMRI allows the assessment of intraoperative developments with the best available imaging modality and the situationadapted presentation of this information with the use of neuronavigation. A high degree of professional competence of all OR staff is required to operate such a complex technique. Increasing system integration and new imaging modalities of the high-field scanners open new perspectives on the efficiency and the risk profile of the surgical procedure. The analysis of our own data shows a significant increase in the rate of complete tumor resection for gliomas, as well as the pituitary adenomas.

\section{Einleitung}

Die Resektion von Tumoren des Gehirns stellt für den Chirurgen eine besondere Herausforderung dar. Die Entfernung vom gesunden Hirngewebe führt unweigerlich zu einer Beeinträchtigung der Gehirnfunktion, welche sehr unterschiedlich ausgeprägt sein und auch im verschiedenem Maße kompensiert werden kann. Häufig kann sogar noch durch Tumor infiltriertes Gewebe zum großen Teil funktionstüchtig sein. Daher ist es in der Neurochirurgie essenziell, die Tumorresektion auf das unmittelbar erkrankte Gewebe zu beschränken. Häufig ist aber auch dieses Ziel ohne ein funk-

OP-JOURNAL 2014; 30: 160-163

(c) Georg Thieme Verlag KG Stuttgart · New York DOI http://dx.doi.org/10.1055/s-0034-1383390

tionelles Defizit nicht zu erreichen. Erschwert wird diese Aufgabe dadurch, dass sich Gehirntumoren auch unter Einsatz des Operationsmikroskops nur schlecht vom Marklager des Gehirns unterscheiden lassen. Häufig sind auch die Tumorgrenzen aufgrund des infiltrativen Tumorwachstums sehr unscharf. Andererseits ist eine vollständige Tumorresektion einer der wichtigsten prognostischen Faktoren.

Die beste Methode, einen Gehirntumor darzustellen, bietet die Magnetresonanztomografie (MRT). Um die hier gewonnenen Informationen optimal während der Operation zu nutzen, wurde die Neuronavigation entwickelt. Sie erlaubt es dem Operateur, die Bildinformation auf den Operationssitus zu übertragen. Diese Information kann entweder auf dem Display der Neuronavigation angezeigt oder in das Okular des Operationsmikroskops eingeblendet werden (siehe Abb. 1) [1]. Die Limitation dieser Methode liegt aber darin, dass während einer Tumorresektion die intrakranielle Anatomie verändert wird und die präoperativ gewonnenen MRT-Daten unter dem Eingriff nicht mehr der Realität entsprechen. Die intraoperative MRT (iMRT) ermöglicht durch Akquisition eines neuen Datensatzes, die intraoperativ veränderte Situation widerzuspiegeln [2]. Seit der Entwicklung der ersten Installationen Ende der 90er-Jahre konnten mehrere Studien den Nutzen des hohen technischen, materiellen und personellen Einsatzes demonstrieren [3-6].

Durch die enorme Weiterentwicklung der diagnostischen Magnetresonanztomografie wurden auch die Möglichkeiten und Einsatzgebiete ihrer intraoperativen Anwendung erheblich erweitert. Scanner mit höheren Feldstärken können nicht nur immer feinere anatomische Strukturen auflösen, sondern auch funktionelle Einheiten und metabolische Prozesse darstellen [7,8]. Diese Eigenschaften erlauben es, die neurochirurgischen Operationen am menschlichen Gehirn nicht nur effektiver, sondern auch sicherer und risikoärmer durchzuführen. Darüber hinaus bieten moderne Installationen in integrierten Hybrid-OPs einen MRT-kompatiblen Anästhesiearbeitsplatz und einen einfachen und effizienten Transfer des Patienten zwischen dem OP-Bereich und dem Scanner, die den Hygienestandards eines neurochirurgischen OPs entsprechen und eine Gefährdung des Patienten verhindern.

\section{Aufbau}

Im Laufe der Zeit haben sich mehrere Lösungen für die Implementation der intraoperativen MRT entwickelt. Unterscheiden kann man Installationen mit Niederfeldscannern mit Magnetfeldstärken bis 0,5 T. Diese Scanner bieten die 

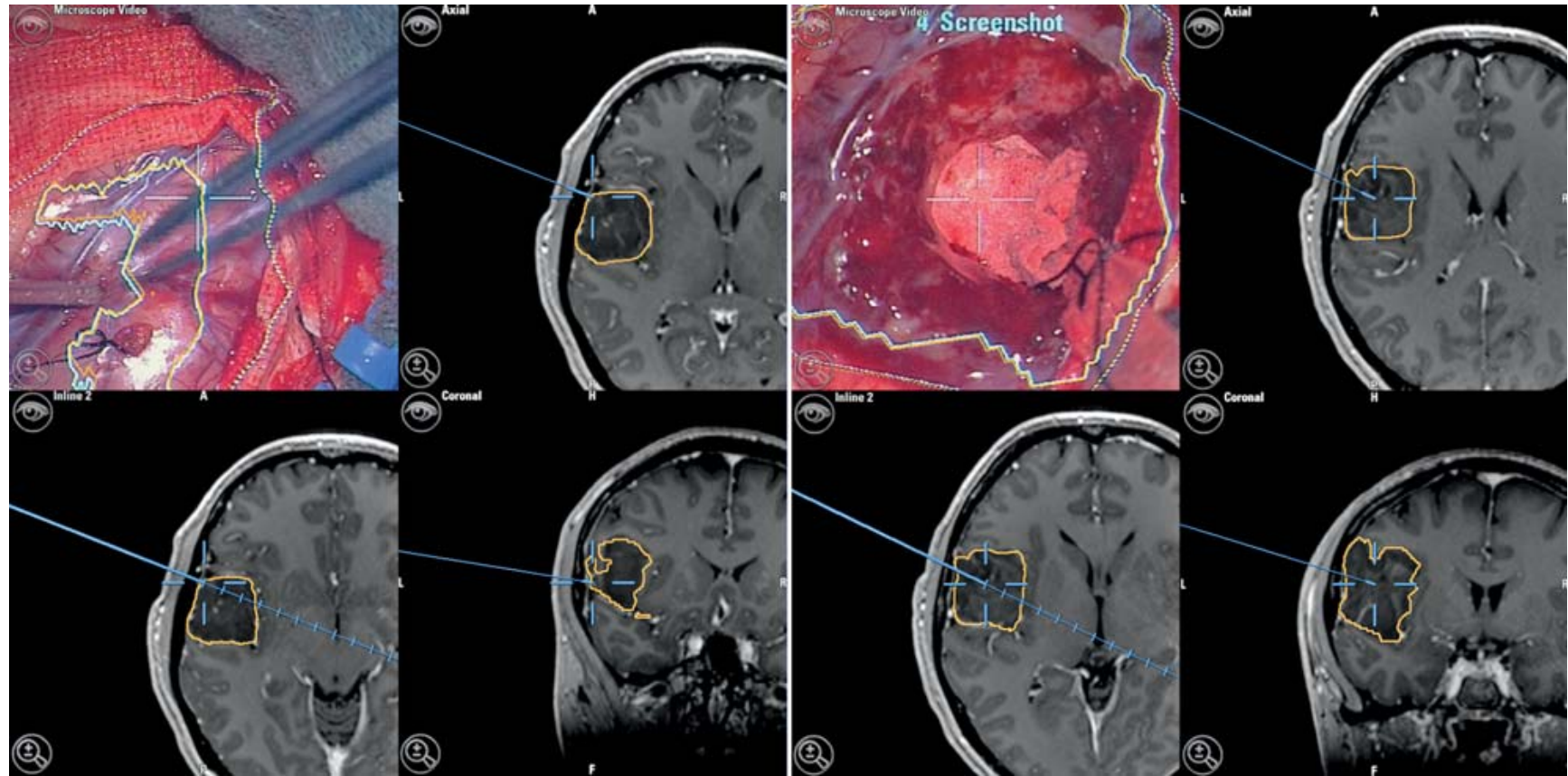

Abb. 1 Darstellung des Operationssitus am Bildschirm der Navigation. Die linke Bildhälfte zeigt den Situs zu Beginn der Tumorresektion. Im Mikroskopbild werden die Tumorgrenzen in der Fokusebene mit durchgezogener Linie und die maximale Tumorausdehnung entlang der optischen Achse mit gestrichelten Linien eingeblendet. Das blaue Fadenkreuz repräsentiert den Fokuspunkt des Mikroskops. Unter der Resektion (rechte Bildhälfte) wird der Fokus in die Tiefe verlagert, was man in den MRT-Aufnahmen gut verfolgen kann.

Möglichkeit einer kompakten Installation, die auch nachträglich in fast jedem Operationssaal möglich ist. Die Scanner sind i.d.R. mobile Geräte, die nicht größer als ein konventioneller C-Bogen sind. Zur Bildakquisition wird der Magnet über dem Kopf des Patienten positioniert und nach der Messung wieder entfernt. Der wesentliche Nachteil dieser Lösung ist die im Vergleich zu diagnostischen MRT-Aufnahmen deutlich geringere Bildqualität, ein eingeschränkter Untersuchungsbereich und die Beschränkung auf konventionelle morphologische Bildgebung. Im Gegensatz dazu stehen die Installationen mit Hochfeldscannern mit Feldstärken ab 1,5 T. Für diese Installationen werden i.d.R. leicht modifizierte konventionelle Scanner verwendet. Sie bieten den Vorteil der Bildgebung in üblicher diagnostischer Qualität mit einem breiten Spektrum an verfügbaren Bildgebungsmodalitäten. Diese Installationen können entweder für eine dezidierte intraoperative Anwendung oder auch für eine kombinierte diagnostische und intraoperative Anwendung ausgelegt sein. Davon abhängig kann der Scanner entweder direkt im Operationssaal installiert sein (siehe Abb. 2) oder sich im Nebenraum befinden. Üblicherweise wird zur Bildgebung der Patient in den Scanner transferiert. Dabei verbleibt der offene Kopf in einer rigiden, für die mikrochirurgische Operationstechnik unerlässlichen Halterung fixiert. In diese
Halterung ist die MR-Spule integriert, um die optimale Bildqualität zu erhalten. Zur Bildakquisition wird der Operationssitus steril abgedeckt und der Patient in den Scanner transferiert. Nach der Messung wird der Patient zurück in den OPBereich transferiert. Die unsterile Abdeckung wird entfernt und der Situs neu

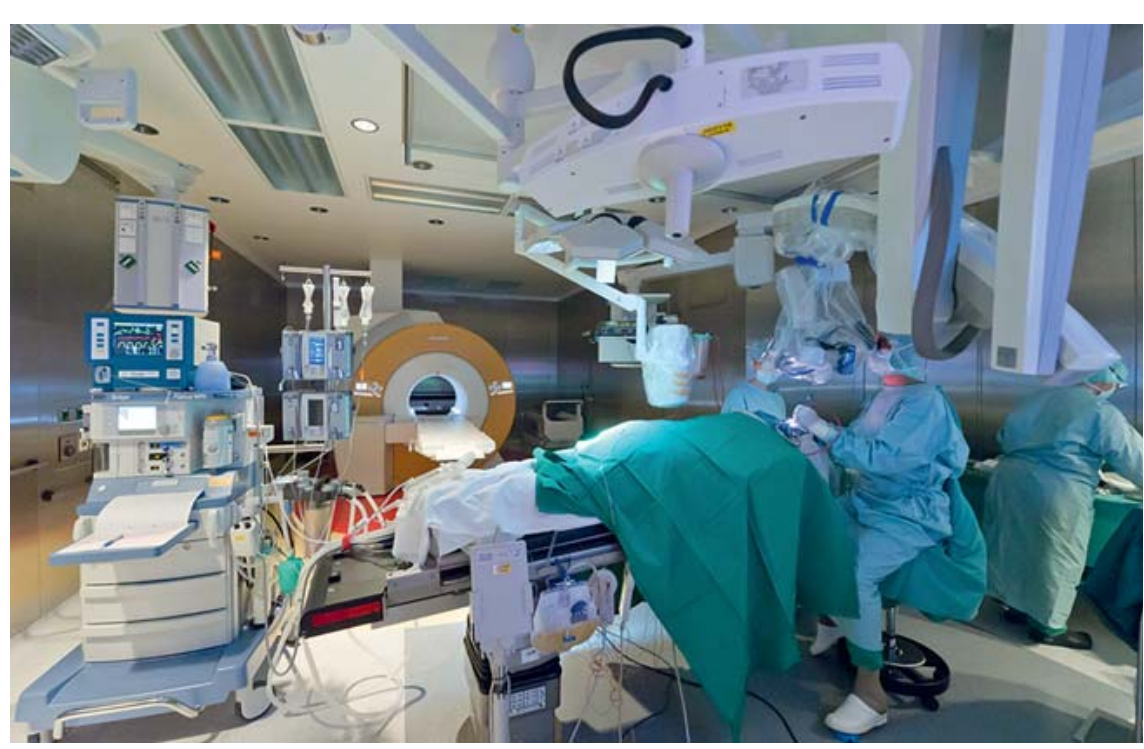

Abb. 2 Integrierter neurochirurgischer Operationssaal „Brainsuite ${ }^{\circledR \text { “ }}$ während eines intrakraniellen Eingriffs. Der Patient befindet sich in OP-Position. Instrumententische sind ganz rechts im Bild. Die Operation erfolgt unter Einsatz des deckenmontierten OP-Mikroskops. Zum Scan wird der Patient auf dem angepassten OP-Tisch um $90^{\circ}$ zum Scanner (im Hintergrund zu sehen) geschwenkt. Der Kopf ist in einer MR-kompatiblen Halterung mit integrierter 8-Kanal-Spule fixiert. Links im Bild findet sich der MR-kompatible Anästhesiearbeitsplatz. Die integrierte Neuronavigation verfügt über eine deckenmontierte Infrarotstereokamera (oben im Bild) und einen ebenfalls deckenmontierten berührungsempfindlichen Bedienmonitor. 


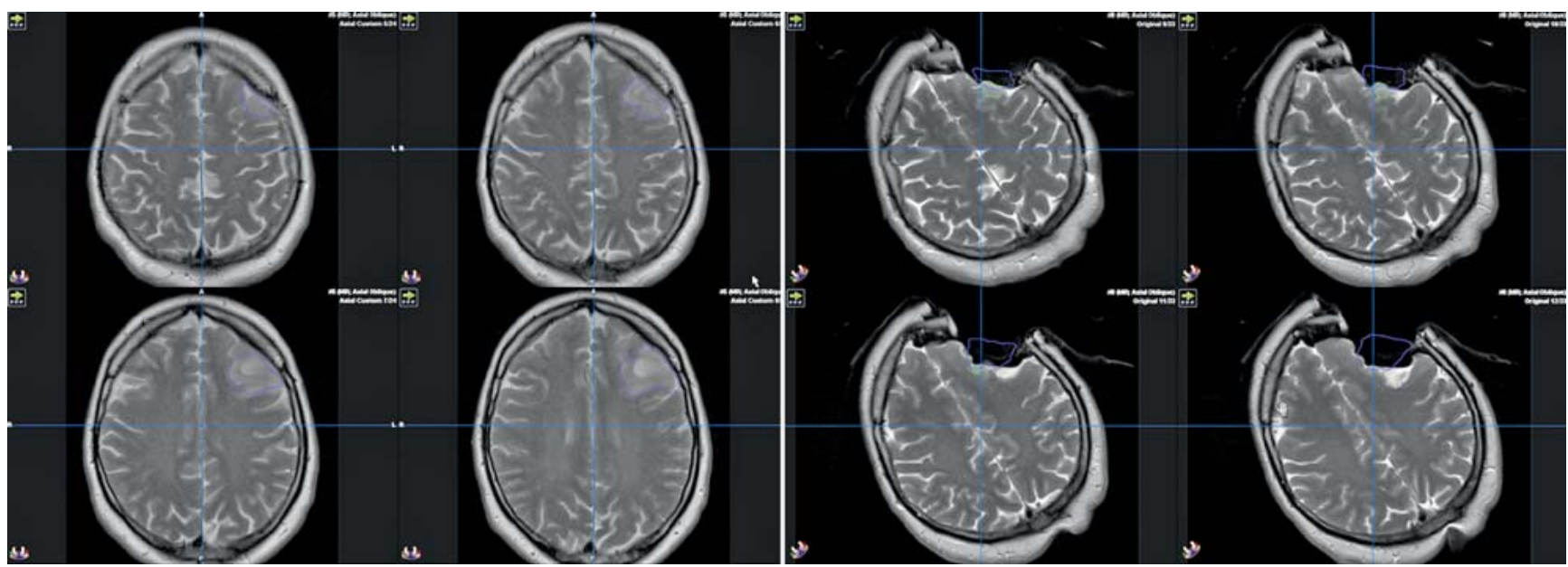

Abb. 3 Präoperative T2-gewichtete axiale Sequenz. Die linke Bildhälfte zeigt das präoperative Planungsbild mit violett markiertem Tumor, der sich vom umliegenden gesunden Hirngewebe durch stärkeres T2-Signal abhebt, wobei die Grenzen sehr unscharf sind. Die rechte Bildhälfte zeigt die intraoperative Situation bei dem gleichen Patienten. Durch Resektion von Tumorgewebe und Verlust von Liquor kommt es zum Brainshift. Der grün markierte Tumorrest liegt außerhalb der ursprünglich eingezeichneten Tumorkontur. Bei direktem Vergleich zeigt sich, dass sich dieser Bereich ursprünglich innerhalb der Kontur befand. Da sich der Tumor in unmittelbarer Nachbarschaft des motorischen Sprachzentrums (Broca) sowie des primär motorischen Kortexes befindet, ist eine genaue Einhaltung der Resektionsgrenzen unerlässlich.

werden. Dafür ist ein MR-kompatibler Anästhesiearbeitsplatz erforderlich.

Die eigentliche Operation erfolgt hingegen außerhalb des Magnetfelds und kann daher mit konventionellem Instrumentarium durchgeführt werden. Es muss allerdings streng darauf geachtet werden, dass keine magnetischen Gegenstände in die Nähe des Magnetfelds geraten. Bei den Scannerinstallationen im OP-Saal ist also zusätzlich zu einer Kontrolle vor dem Patiententransfer, bei der sichergestellt werden muss, dass am Patienten keine magnetischen Instrumente verblieben sind, auch während des gesamten OP-Betriebs darauf zu achten [9]. Ein speziell geschultes Personal ist für den Betrieb eines OP-Saals mit iMRT unerlässlich.

\section{Behandlung}

Die Durchführung einer Hirntumorresektion in einem modernen Hybrid-OP unterscheidet sich nur wenig von der Durchführung in einem Standard-OP. Der narkotisierte Patient wird auf dem OP-Tisch gelagert und Kopf wird in der Kopfhalterung fixiert. Hier besteht im Vergleich zu einer konventionellen Operation eine gewisse Einschränkung der Bewegungsfreiheit, da der Kopf mit Kopfhalterung später in die Bohrung des Scanners hineinpassen muss. Die Navigation kann entweder über die Hautoberfläche oder Fiducials registriert werden. Darüber hinaus hat man bei schwierigen Lagerungen auch die Möglichkeit einer automatischen Registrierung durch Anfertigung eines Registrie- rungsscanns. Diese Möglichkeit bietet die optimale Navigationsgenauigkeit, erfordert aber einen zusätzlichen MRTScan. Die eigentliche Operation erfolgt dann wie in einem konventionellen Operationssaal. Wenn der Operateur den Eindruck hat, den Tumor vollständig entfernt zu haben oder die Navigationsgenauigkeit aufgrund von Brainshift ungenügend ist, wird eine MRT-Untersuchung durchgeführt. Mit den neu gewonnen Daten kann die Navigation aktualisiert werden. Wenn nötig, kann dann der evtl. verbliebene Tumorrest sicher angegangen werden. Die Schritte können dabei nach Bedarf mehrfach wiederholt werden (siehe Abb. $\mathbf{3}$ und 4).

Die Verwendung von zusätzlichen Hilfsmitteln wie Fluoreszenzmikroskopie, intraoperative Sonografie oder Elektrophysiologie ist dabei ohne Einschränkung möglich $[10,11]$. Vor allem das intraoperative elektrophysiologische Monitoring gewinnt zunehmend an Bedeutung. Mithilfe dieses Verfahrens ist es möglich, gewisse Gehirnfunktionen auch bei narkotisiertem Patienten kontinuierlich während der Operation zu überwachen [12]. Das ergänzt sich mit der iMRT besonders gut, weil man dadurch auch die intraoperativen Veränderungen in der Elektrophysiologie morphologisch verifizieren kann. Vor allem das Monitoring des primär motorischen Kortexes und der Pyramidenbahn gehört in unserer Klinik zum Standard bei Eingriffen in der Nähe dieser Strukturen. Dabei kann das Monitoring während des mehrstündigen Eingriffs eine Amplitudenminderung der motorisch evozierten Potenzia- le (MEP) aufweisen und auf die Gefahr einer postoperativen Parese hindeuten. Allerdings kann diese Amplitudenminderung mehrere Ursachen haben und nicht immer liegt es daran, dass die Strukturen durch die Resektion geschädigt werden. Um zu entscheiden, ob mit der Resektion ohne hohes Risiko einer bleibenden Parese fortgefahren werden kann, ist die iMRT hilfreich.

Von über 800 in unserer Einrichtung durchgeführten Operationen mit iMRT waren es in $55 \%$ Operationen bei glialen Tumoren, 24\% Tumoren der Hypophysenregion, $10 \%$ nichtgliale Tumoren, $9 \%$ vaskuläre Malformationen, $2 \%$ epilepsiechirurgische Eingriffe und $8 \%$ sonstige Operationen. In einer eigenen Auswertung von 158 Patienten mit primären ZNS-Tumoren führte der Einsatz der iMRT bei 106 (67,1\%) Patienten zur Identifikation von einem Tumorrest und zu einer Fortsetzung der Resektion. Bei 51 von diesen Patienten konnte dadurch der Tumor vollständig reseziert werden, was einer Steigerung der Rate an kompletten Resektionen um 32,2\% entspricht. Bei transsphenoidalen Operationen an Hypophysentumoren konnte durch Identifikation des Tumorrests im intraoperativen MRT in 27,9\% der Eingriffe eine Vervollständigung der Tumorresektion erreicht werden [13].

\section{Zusammenfassung}

Durch überzeugende klinische Ergebnisse und technische Innovationen, die Installation und Betrieb deutlich erleichtern konnten, fand die iMRT in der Neu- 

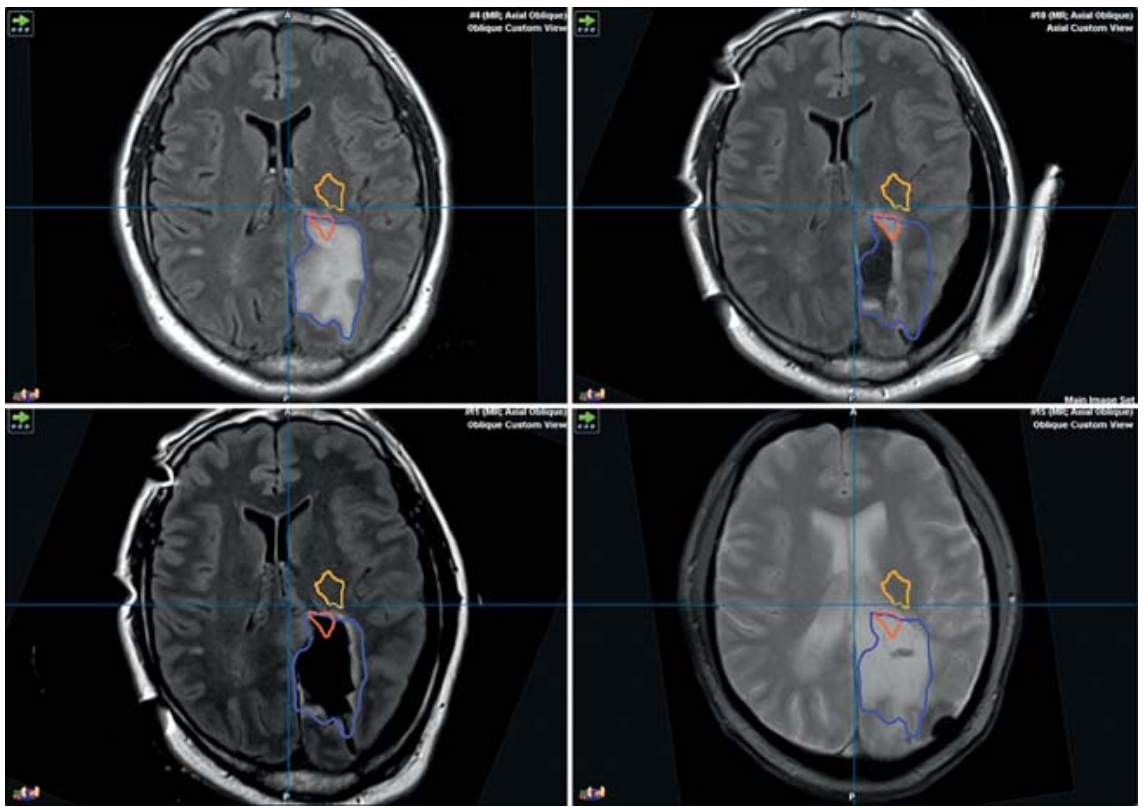

Abb.4 Intraoperative Bildgebung während der Resektion eines ausgedehnten Oligoastrozytoms WHO-Grad III. Links oben ist das präoperative Bild. Die blaue Linie zeigt die geplanten Resektionsgrenzen. Die gelbe Linie markiert die Pyramidenbahn. Rechts oben ist die 1. intraoperative Aufnahme. Diese zeigt eine annähernd vollständige Tumorresektion. Rot eingezeichnet ist ein zu resezierender Tumorrest, welcher in unmittelbarer Nähe der Pyramidenbahn belassen wurde, weil es während der Resektion zu einem deutlichen Brainshift gekommen war. Er ist deutlich an dem Spalt zwischen Kortexoberfläche und Schädelkalotte links parietal sowie dem gesunden Hirnparenchym, welches sich nun innerhalb der initialen blauen Markierung befindet, zu erkennen. Links unten ist die gleiche Schicht am Ende der Operation. Man kann die vollständige Entfernung des Tumors einschließlich des kleinen Restes an der Pyramidenbahn gut erkennen. Rechts unten ist ein Verlaufs-MRT 3 Jahre nach der Operation.

rochirurgie in den letzten Jahren eine zunehmende Verbreitung. Das primäre Einsatzgebiet der iMRT ist bei der Operation glialer Tumoren des Gehirns. Mit der iMRT kann nicht nur die intraoperative Resektionskontrolle erfolgen, die in vielen Fällen zu einer Fortführung der Resektion führt, sie kann auch zur Validierung des intraoperativen elektrophysiologischen Monitorings eingesetzt werden. Darüber hinaus sind durch neue bildgebende Modalitäten wie Diffusionsund Perfusionsbildgebung neue Einblicke in die Tumorbiologie auf der einen und Gehirnfunktion auf der anderen Seite möglich geworden, die u.U. zu einer neuen Betrachtung des Operationsziels führen können. In der Gliomchirurgie konnten mehrere Studien einen günstigen Einfluss der iMRT auf das Ausmaß der Tumorresektion und auch auf das Outcome der Patienten zeigen. Zunehmend findet die iMRT auch Einsatz in der transsphenoidalen Resektion der Hypophysenadenome. Auch hier sind Studien, die eine höhere Rate an vollständigen Tumorresektionen zeigen, publiziert worden. Mit der Weiterentwick- lung der MRT-Technik ergeben sich in vielen Bereichen neue Möglichkeiten der Bildgebung mit Darstellung von funktionellen Arealen und Faserbahnen, welche dem Chirurgen ein risikooptimiertes Vorgehen und eine geringere Morbidität ermöglichen. Die iMRT ist ein sehr nützliches Verfahren in der Behandlung ausgewählter Tumoren des Gehirns. Aufgrund von hohen Investitionskosten, einem nicht unerheblichen personellen Aufwand und der erforderlichen Expertise bleibt sie wohl ein Verfahren, das nur spezialisierten Zentren vorbehalten sein wird.

\section{Literatur}

${ }^{1}$ Wirtz CR, Kunze S. Neuronavigation: Computerassistierte Neurochirurgie. Dtsch Arztebl 1998; 95: A-2384

2 Tronnier VM, Wirtz CR, Knauth M et al. Intraoperative diagnostic and interventional magnetic resonance imaging in neurosurgery. Neurosurgery 1997; 40: 891-900

3 Senft C, Bink A, Franz K et al. Intraoperative MRI guidance and extent of resection in glioma surgery: a randomised, controlled trial. Lancet Oncol 2011; 12: 997-1003

${ }^{4}$ Roder C, Bisdas S, Ebner FH et al. Maximizing the extent of resection and survival benefit of patients in glioblastoma surgery: highfield iMRI versus conventional and 5-ALA-assisted surgery. Eur J Surg Oncol 2014; 40: 297-304

${ }^{5}$ Schmidt T, König R, Hlavac $M$ et al. Lows and highs: 15 years of development in intraoperative magnetic resonance imaging. Acta Neurochir Suppl 2011; 109: 17-20

${ }^{6}$ Knauth M, Wirtz CR, Tronnier VM et al. Intraoperative MR imaging increases the extent of tumor resection in patients with high-grade gliomas. AJNR Am J Neuroradiol 1999; 20: 1642-1646

7 Stadlbauer A, Nimsky C, Gruber S et al. Changes in fiber integrity, diffusivity, and metabolism of the pyramidal tract adjacent to gliomas: a quantitative diffusion tensor fiber tracking and MR spectroscopic imaging study. AJNR Am J Neuroradiol 2007; 28: 462469

8 Nimsky C, Ganslandt O, Merhof D et al. Intraoperative visualization of the pyramidal tract by diffusion-tensor-imaging-based fiber tracking. Neuroimage 2006; 30: 1219-1229

${ }^{9}$ Hall WA, Liu H, Martin AJ et al. Safety, Efficacy, and functionality of high-field strength interventional magnetic resonance imaging for neurosurgery. Neurosurgery 2000; 46: 632 641

10 Coburger J, Engelke J, Scheuerle A et al. Tumor detection with 5-aminolevulinic acid fluorescence and Gd-DTPA-enhanced intraoperative MRI at the border of contrast-enhancing lesions: a prospective study based on histopathological assessment. Neurosurg Focus 2014; 36: E3

${ }^{11}$ Coburger J, König RW, Scheuerle A et al. Navigated high frequency ultrasound: description of technique and clinical comparison with conventional intracranial ultrasound. World Neurosurg 2014; 82: 366-375

12 Simon $M$, Neuloh $G$ von Lehe $M$ et al. Insular gliomas: the case for surgical management. J Neurosurg 2009; 110: 685-695

13 Coburger J, König R, Seitz K et al. Determining the utility of intraoperative magnetic resonance imaging for transsphenoidal surgery: a retrospective study. J Neurosurg 2014: 120: 346-356

Prof. Dr. med. Rainer Wirtz Ärztlicher Direktor

Klinik für Neurochirurgie Universität Ulm Albert-Einstein-Allee 23 89081 Ulm

\section{Dr. med. Michal Hlavac Oberarzt} PD Dr. med. Ralph König Oberarzt

Neurochirurgische Klinik der Universität Ulm Bezirkskliniken Schwaben Ludwig-Heilmeyer-Straße 2 89312 Günzburg

michal.hlavac@bkh-guenzburg.de ralph.koenig@bkh-guenzburg.de 